\title{
La quinua (Chenopodium quinoa Willd), un tesoro andino para el mundo
}

\author{
José Delatorre-Herrera $^{1 *}$, M. Sánchez, I. Delfino, M.I. Oliva ${ }^{1}$
}

\section{Introducción}

Según el INE (2007), la región con más superficie en Chile es Tarapacá, con 1.357 ha de quinua, la que en 2009 se reduce a 250 ha, mostrando una drástica disminución del $81,5 \%$, lo anterior es consecuencia de las inestables condiciones ambientales del Altiplano, lo que se traduce en una disminución de las lluvias (Arenas, 2011). El principal productor de quinua del mundo es Bolivia con 64.789 hectáreas, 38.257 toneladas totales y $590 \mathrm{~kg} \mathrm{ha}^{-1}$ de grano promedio (Ramos y García, 2010; FAO, 2011).

Los agricultores que cultivan quinua manejan sus cultivos con un nivel tecnológico que los hace poco competitivos, sus rendimientos esperados no superan los 600 a $900 \mathrm{~kg} \mathrm{ha}^{-1}$ en época lluviosa y de $60 \mathrm{~kg} \mathrm{ha}^{-1}$ en períodos de sequía (Delatorre et al., 2008). Esto requiere de urgentes innovaciones tecnológicas que permitan mejoras en el proceso productivo, como variedades mejoradas a partir de su propio germoplasma, sistemas de riego, maquinarias de poscosecha, entre otros. Por otro lado, gran parte de la producción de quinua del Altiplano chileno se transa en el mercado informal, ya sea en la feria interfronteriza o por intermedio de compradores bolivianos, los que pagan entre US\$ 80 y US\$ 120 por quintal español (46 kg).

\section{Variedades}

En el Altiplano de Chile existe una alta variabilidad en la quinua que es cultivada (Fuentes 2009). Muchas veces las semillas que se emplean han sido seleccionadas por fenotipos por los propios agricultores (Arar et al., 2011). Por ejemplo, el color de pericarpio del grano que puede variar entre blanco, amarillo, rojo y negro. También se cultivan como mezcla de diferentes fenotipos las que presentan variaciones como el color de las hojas, color de la panoja, altura, color de pericarpio de la semilla, entre otras; sin embargo, este procedimiento les confiere a los agricultores un grado de seguridad ante cambios climáticos inesperados, como sequías, heladas, entre otros (Fuentes, 2009). La selección de los caracteres que se quiere mejorar genéticamente dependerá de los factores deseables en el proceso de producción, industrialización y consumo. Por ejemplo, un problema tecnológico que se presenta es la desuniformidad en la maduración de los ecotipos locales, lo que afecta la cosecha, ya que solo se puede realizar manual. Esto ocurre porque no existen variedades, por lo que cada individuo es diferente dentro del mismo ecotipo en sus características morfológicas. Lo anterior se refuerza cuando se observa la duración del ciclo del cultivo, existiendo plantas con ciclos de 150 hasta 210 días desde siembra a cosecha (Delatorre et al., 2008).

El proceso de mejoramiento de la quinua implica utilizar técnicas de mejoramiento adecuadas para plantas autógamas, lo que además requiere tomar precauciones para evitar una fecundación cruzada (encapuchamiento de plantas, aislamiento de campos). De esta forma, con el método de selección no se crea un genotipo nuevo, si no se selecciona de un grupo heterogénico de plantas un genotipo ya existente, que presenta los caracteres deseados (ERPE, 2001).

Lo importante es que en el material se presente suficiente variación genética para poder realizar exitosamente una selección. Entre 2005 y 2007 en el marco del proyecto FIA-PI-C-20041-A-079, la Universidad Arturo Prat inició un proceso de selección en el Altiplano chileno. Las plantas se seleccionaron y cosecharon a partir de su fenotipo (Poehlman y Allen, 2003) enfocado principalmente en color de panoja identificando

\footnotetext{
1 Universidad Arturo Prat, Iquique, Chile.

* Autor por correspondencia: jose.delatorre@unap.cl
} 
dos ecotipos, blanca (Jank'u) y Roja (Pandela). Las líneas seleccionadas, además de la caracterización morfológica, fueron analizadas genéticamente mediante marcadores moleculares del tipo RAPD. Una vez analizadas las distancias genéticas, las semillas se mezclaron formando un nuevo grupo de plantas más homogéneas en características como altura de plantas y contenido de saponinas, pero manteniendo una variabilidad, este procedimiento se repitió durante tres ciclos de selección (Informe 4, proyecto: FIA-PI-C-2004-1-A-079, 2009). El grupo seleccionado por medio de este procedimiento es normalmente uniforme en cuanto a caracteres cualitativos que presentan herencia genética simple, tal como se plantea en relación con el color de la panoja y altura de planta antes del momento de la cosecha. Los resultados de esta selección muestra un incremento en la altura de la planta (AP) tanto para el ecotipo blanco como para el ecotipo rojo en $10 \mathrm{~cm}$, otra variable que aumentó fue el peso de los 100 granos (P100G), lo que se traduce en un aumento de la producción de granos de la planta; variables como el contenido de saponina disminuyeron en los dos ecotipos pasando de quinuas semidulces a dulces; sin embargo, se ha detectado que el rendimiento disminuye en inversa proporción respecto del contenido de saponinas, lo que sugiere que este compuesto está ligado a algún tipo de resistencia de la planta o a productividad (Informe 4, proyecto: FIA-PI-C-2004-1-A-079, 2009).

\section{Suelos y Fertilidad}

Respecto del suelo, estudios realizados por Delatorre (2008) muestran que la fertilidad de los suelos del Altiplano, específicamente en el sector de Cruz Pampa, Vilacollo y Po'ko loma es muy pobre, no alcanzando las unidades mínimas que permiten obtener y/o garantizar un buen rendimiento. Esto se ve agravado por el $\mathrm{pH}$, el que fluctúa entre 7,7 y 8,8 , limitando la absorción de los elementos que pudiesen estar disponibles.

$\mathrm{Al}$ analizar los macroelementos, solo el $\mathrm{K}$ se encuentra en concentraciones adecuadas $(255 \mathrm{mg}$ $\mathrm{kg}^{-1}$ ), en tanto que nitrógeno y fósforo se clasifican como muy bajos, 17 y $7 \mathrm{mg} \mathrm{kg}^{-1}$, respectivamente (Delatorre, 2008).

La fertilidad de los suelos está relacionada con las prácticas que se empleen para su recuperación. En este caso, la única actividad que realizan los agricultores es la aplicación de estiércol obtenido de sus animales. El guano que utilizan posee buenas características físico-químicas, con pH de 7,4, una C.E de $1,8 \mathrm{dS} \mathrm{m}{ }^{-1}, 1,6 \%$ de nitrógeno y $0,82 \%$ de fósforo; sin embargo, las cantidades aplicadas son bajas.

Aun así, considerando las condiciones anteriores, la quinua es capaz de producir, aunque sus producciones no sobrepasan los $600 \mathrm{~kg} \mathrm{ha}^{-1}$ en el Altiplano y $800 \mathrm{~kg} \mathrm{ha}^{-1}$ con aguas altamente contaminadas y salinas. Para mejorar los rendimientos se ha determinado un programa de fertilización base para suelos pobres que permita alcanzar rendimientos desde los $1.600 \mathrm{~kg} \mathrm{ha}^{-1}$ (PIAT, 2011), este considera aplicar entre 120 y190 unidades de nitrógeno, 60 a 70 unidades $\mathrm{P}$ y $20.000 \mathrm{~kg}$ de guano por ha ${ }^{-1}$.

Sumado a la deficitaria fertilidad de los suelos como factor que disminuye los rendimientos se debe agregar la falta de precipitaciones, la que en los últimos años se ha hecho cada vez más crítica, tal como lo indica Arenas (2010), quien señala que las precipitaciones son variables y muchas veces no son suficientes para lograr una óptima cosecha de la quinua en el Altiplano chileno.

\section{Riego en Quinua}

El Altiplano chileno se encuentra a una altitud de $3.700 \mathrm{msnm}$ aproximadamente. Las precipitaciones se presentan en la época estival entre noviembre y marzo, con concentraciones en diciembre, pero esta condición ha cambiado en los últimos años, concentrándose en enero y febrero y desplazándose hasta mayo, acompañado todo esto por una disminución de más del 50\% del agua lluvia caída anualmente en los últimos años (Arenas, 2011). La quinua, cultivo de grano que crece en condiciones de Altiplano, se caracteriza por su adaptabilidad a condiciones desfavorables de suelo y clima (Geerts et al., 2008), posee varios mecanismos relacionados con la tolerancia y escape a la sequía. Este escape a la sequía se muestra como una prolongación en el ciclo de crecimiento en respuesta a la falta de agua en los períodos vegetativos tempranos y provoca maduración temprana en los estados tardíos de crecimiento (Jacobsen et al., 2003a).

Pese a estos mecanismo de tolerancia a la falta de agua, el rendimiento de la quinua se ve perjudicado, alcanzando en el Altiplano de la Región de Tarapacá solo 6 a $900 \mathrm{~kg} \mathrm{ha}^{-1}$ con precipitaciones 
adecuadas, según lo investigado en el proyecto FIA-PI-C-2004-1-A-079 (Delatorre et al., 2009), esto debido a la escasez del recurso hídrico en los primeros meses de crecimiento del cultivo (Bosque Sánchez et al., 2003).

A pesar de que los suelos de Cariquima presentan una baja humedad aprovechable (4,6\%), los agricultores siembran la quinua en terrenos donde han acumulado agua en el suelo durante dos años. De esta manera, el agricultor siembra a partir de agosto y como fecha límite noviembre; los valores de humedad de suelo son muy cercanos a capacidad de campo (9,3\%) (Delatorre et al. 2008). Para ello deposita las semillas en el suelo húmedo y espera su germinación solamente con la humedad del suelo. La quinua debe crecer con esta agua hasta que se reciban las lluvias, las que normalmente caen en diciembre. El agricultor en la medida en que retrasa la época de siembra, está más expuesto a perder su cosecha debido a las heladas que caen en enero $\left(-6 C^{\circ}\right)$, y que la quinua es capaz de soportar en un estado fenológico avanzado (posantesis).

Sin duda que una de las formas de asegurar el riego es mediante la aplicación de agua controlada en los estados sensibles de crecimiento, con lo que pueden aumentar los rendimientos significativamente o lograr rendimientos en años con déficit de precipitación (García, 2003; García et al., 2003). Tal como se realizó en una experiencia en la localidad de Vilacollo, donde tanto la siembra como los estados más críticos (floración y llenado de grano) fueron suplementados con un riego (Lanino, et al., 2008).

\section{Manejo de la Cosecha y Poscosecha}

En el Altiplano la cosecha tradicional de quinua es totalmente manual, es habitual el arranque de plantas, las que surgen con raíces, trayendo tierra y piedras, que al momento de la trilla se mezcla con el grano desmejorando la calidad. Luego, las panojas son almacenadas en parvas, el objetivo de la formación de estas parvas es evitar que la cosecha se dañe. Para esto se mantienen en proceso de secado por aproximadamente dos semanas hasta que los granos tengan la humedad conveniente para la trilla. Las plantas son transportadas a eras y se realiza la trilla semimecanizada, donde se provoca el roce de las panojas mediante el paso de neumáticos. Nieto y Soria (1991) mencionan que existe en campo un $16 \%$ de pérdida en poscosecha.
En Bolivia y Ecuador se realiza la siega de las plantas con una hoz, lo que provoca una disminución de grano contaminado, menor pérdida y tiempo de cosecha. Un sistema mejorado de cosecha de quinua consiste en la utilización de trilladoras estacionarias. En Perú, Bolivia y Ecuador se han utilizado varios modelos de trilladoras de cereales, los cuales han sido adaptados para la trilla de quinua; en otros casos se han creado prototipos específicos para quinua, todos con aceptable efectividad y rendimiento (Tapia, 1990; Nieto y Soria, 1991; Nieto y Vimos, 1992; Nieto et al., 1986).

Las semillas poseen la presencia de factores antinutricionales que inciden directamente en la asimilación y/o en el valor nutritivo de los alimentos y corresponden a taninos, inhibidores de proteasa, ácido fítico, saponinas, sapogeninas, fracción de escualeno, terpenoides, ácidos grasos oxidados, oxalatos y sales de magnesio. De estas, las saponinas que son glicoalcaloides que se encuentran en la cubierta del grano de quinua y que le dan un sabor amargo que impide su consumo directo (Oliva, 2008). Si estos compuestos no se eliminan totalmente aparecen en los alimentos preparados en base a quinua y podrían presentar sabores astringentes, jabonosos, picantes o rancios (Bacigalupo y Tapia, 1990; Ruales 1992a; Ruales 1992b).

En la mayoría de las áreas de producción tradicional de quinua, en la zona andina, la eliminación de saponina de los granos de quinua se hace tostando el grano, frotando para eliminar la cascarilla quemada, para finalmente efectuar un lavado manual, proceso que permite trabajar solo con pequeños volúmenes (Oliva, 2008).

El escarificado por método seco somete a los granos de quinua a un proceso combinado de efecto abrasivo y golpeado. En una máquina escarificadora tiene una eficiencia del $95 \%$ y los contenidos de saponina en el producto final oscilan entre el $0,04 \%$ y $0,25 \%$, dependiendo de la variedad utilizada como materia prima. Por otra parte, el método húmedo para la desaponificación del grano consiste en someter los granos a un proceso de lavado y turbulencia, en agua circulante o fija en el recipiente de lavado; la saponina se elimina en el agua de lavado, posteriormente se somete el grano a un proceso de secado, obteniéndose porcentajes de saponinas similares a la escarificación en seco (Oliva, 2008); sin embargo, se corre el riesgo de la que las semillas germinen, lo que afectará la calidad nutricional de la quinua. 


\section{Conclusiones}

Las bajas condiciones de fertilidad de los suelos del Altiplano y la poca seguridad de riego limitan fuertemente la producción de quinua, razones por las cuales se deben corregir los suelos desde el punto de vista de la fertilidad con aplicación de materia orgánica y fertilizantes nitrogenados y fosfatados. De igual forma se requiere considerar obras de riego que aseguren una adecuada producción y que permita tener una oferta permanente en el tiempo.

\section{Bibliografía}

Arar, Matthiew, A.; Gómez, E.; Choque, J.; Arenas, J.; DelatorreHerrera, J. y Sánchez, M.

2009. La historia a dos voces de las cooperativas de quinua en la región de Tarapacá: Estrategias y Desafíos. Revista Geográfica de Valparaíso. № 42: 112-123.

Arenas J.

2011. Observatorio del clima para el año 2010 en el sector Vilacollo, comuna de Colchane, provincia del Tamarugal, región de Tarapacá. Universidad Arturo Prat. Departamento de Agricultura del Desierto y Biotecnología.

Bacigalupo, A. y Tapia, M..

1990. Potencial agroindustrial de los cultivos andinos subexplotados. En: Tapia M. (ed.). Cultivos Andinos subexplotados y su aporte a la alimentación. FAO. Ediciones Gegra S.A. Santiago, Chile, pp. 136-163.

Bosque Sánchez, H.; Lemeur, R. and Van Damme, P. 2000. Análisis ecofisiológico del cultivo de la quinua (Chenopodium quinoa Willd.) en condiciones de estrés de la sequía y la salinidad. Tropicultura 18: 198-202.

Delatorre, J.

2007. La fertilidad de los suelos y la nutrición de la quinua. FIA-PI-C-2004-1-A-079. 74 p.

Delatorre, J.; Sánchez, M. y Challapa, E. 2008. Manejo cultural de la quinua. E cultivo de la quinua. Revista de Agricultura del Desierto (4): 23-31.

Erpe, Inia, Iica y GTZ.

2001. Taxonomia y morfología de planta. En: Manual de producción de quinua de calidad en el Ecuador. Quito. Disponible en internet: www.ecuarural.gov.ec/ecuagro/ paginas/PRODUCYCS/MANUALES/manual_quinua.htm

Fuentes, F.; Maughan, P.J. and Jellen, E.

2009. Genetic diversity and genetic resources for quinoa breeding. Revista Geográfica de Valparaíso. № 42, 20-33.

García, M.

2003. Agroclimatic Study and Drought Resistance Analysis of Quinoa for an Irrigation Strategy in the Bolivian Altiplano. Dissertationes de Agricultura, Faculty of Applied Biological Sciences, K.U. Leuven, Belgium 556.

García, M.; Raes, D.; Jacobsen, S.E.

2003. Evapotranspiration analysis and irrigation requirements of quinoa (Chenopodium quinoa) in the Bolivian highlands. Agric. Water Manage. 60, 119-134.

Geerts, S.; Raes, D.; Garcia, M.; Vacher, J.; Mamani, R.; Mendoza, J.; Huanca, R.; Morales, B.; Miranda, R.; Usicanqui, J. y

Taboada, C.

2008. Introducing deficit irrigation to stabilize yields of quinoa (Chenopodium quinoa Willd.). Europ. J. Agronomy 28 427-436.
INE.

2007. Compendio Estadístico. Instituto Nacional de Estadística. Chile.

Jacobsen, S.E.; Mujica, A.; Jensen, C.R.

2003a. The resistance of quinoa (Chenopodium quinoa Willd.) to adverse abiotic factors. Food Rev. Int. 19, 99-109. sequía y la salinidad. Tropicultura 18: 198-202.

Lanino, M; Riquelme, A y Arenas, J.

2008. El riego del cultivo de la quinua en el altiplano. Revista de Agricultura del Desierto. № 4: 27-37. ISSN: 0717-2729, 120 pp.

Nieto, C. y Soria, M.

1991. Procesamiento de quinua en Ecuador. Proyecto 3P-850213. Informe final de labores. INIAP-UTA-CIID. Quito, Ecuador, $94 \mathrm{p}$.

Nieto, C. y Vimos, C.

1992. La quinua, cosecha y poscosecha. Algunas experiencias en Ecuador. INIAP, Quito, Ecuador, 43 p. (Boletín divulgativo 224).

Nieto, C., Castillo, C. y Peralta, E.

1986. Guía para la producción de semilla de quinua. INIAP. Quito, Ecuador, 8 p. (Boletín divulgativo 186).

Oliva, María Isabel

2008. Revista de Agricultura del Desierto. № 4: 90-104. ISSN: 0717-2729, $120 \mathrm{pp}$.

PIAT.

2011. Informe Final: Programa de Investigación, Capacitación y Asistencia técnica en Calama. Financiado por Xstrata Lomas Bayas.

Ramos, H. y García, M.

2010. La Agricultura Ecológica de la quinua, una alternativa a los impactos de desertización en el Altiplano Sur. Resumen tercer congreso internacional de la quinua, $147 \mathrm{p}$. Oruro-Bolivia.

Ruales, J. y Nair, B.M.

1992 (a). Quinoa (Chenopodium quinoa Willd), An important andean food crop. Department of Applied Nutrition, University of Lund, Sweeden. Instituto de Investigaciones Tecnológicas, Escuela Politécnica Nacional. Quito, Ecuador, 26 p.

Ruales, J. y Nair, B.M.

1992 (b). Effect of processing on the digestibility of protein and availability of starch in quinoa (Chenopodium quinoa willd) seeds. Department of Applied Nutrition, University of Lund, Sweeden. Escuela Politécnica Nacional. Quito, Ecuador, $23 \mathrm{p}$.

Poehlman, J. and Allen, D.

2003. Mejoramiento genético de las cosechas. Editorial Lumisa. México, 511 pp.

Tapia, M.

1990. Cultivos Andinos subexplotados y su aporte a la alimentación. FAO. Ediciones Gegra S.A. Santiago, Chile, pp. 56-59. 